\title{
Near-complete optic nerve transection by high-pressure air
}

\author{
Soo Won Ko, Jong Seok Lee, Han Sung Choi, Young Gwan Ko, \\ Hoon Pyo Hong
}

Department of Emergency Medicine, Kyung Hee University Hospital, Seoul, Korea

The use of high-pressure air instruments has become more common. Consequently, there have been a number of cases of orbital emphysema caused by contact with high-pressure air. In this case, a 62-year-old male patient visited an emergency medical center after his left eye was shot by an air compressor gun that was used to wash cars. Lacerations were observed in the upper and lower eyelids of his left eye. Radiological examinations revealed orbital emphysema, optic nerve transection, pneumocephalus, and subcutaneous emphysema in the face, neck, shoulder, and mediastinum. Canalicular injury repair was performed, and the emphysema resolved. However, there was near-complete vision loss in the patient's left eye. Because most optic nerve transections occur after a severe disruption in bone structure, pure optic nerve transections without any injury of the bone structure, as in the present case, is extremely rare.

Keywords Optic nerve injuries; Compressed air; Emphysema
Received: 14 May 2016

Revised: 26 May 2016

Accepted: 30 May 2016

Correspondence to: Hoon Pyo Hong Department of Emergency Medicine, Kyung Hee University Hospital, 23 Kyungheedae-ro, Dongdaemun-gu, Seoul 130-872, Korea

E-mail:xrayhong@naver.com

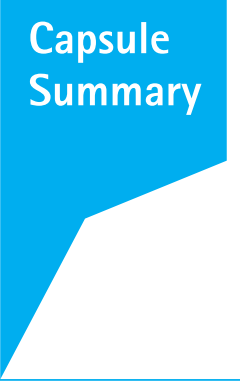

What is already known

Most optic nerve injury follows after severe disruption in bony structure.

What is new in the current study

In our case, high pressure air injury without disruption in bony structure caused optic nerve transection which it ultimately caused permanent visual loss. 


\section{INTRODUCTION}

High-pressure air is now commonly used in many industrial fields as well as in daily life. ${ }^{1-4}$ The use of high-pressure air results in a number of accidents, including orbital emphysema, ${ }^{1-8}$ pneumocephalus, ${ }^{1,2,5,6}$ and orbital fractures. ${ }^{1}$ Most orbital injuries from high-pressure air resolve without any serious sequelae, but, in some cases, visual deterioration may occur. In the literature, severe visual loss is thought to be caused by optic atrophy, which is a consequence of vessel damage to the optic nerve, which then results in decreased blood supply and, ultimately, atrophy of the nerve. ${ }^{8}$ In the present patient, however, a sudden acute increase in intraorbital pressure from high-pressure air caused near-complete optic nerve transection, which was followed by permanent visual loss. To the best of our knowledge, no reports of optic nerve transection from pure soft tissue injury without any fractures exist.

\section{CASE REPORT}

A 62-year-old man visited the emergency medical center after his left eye had been hit and shot by a compressor air gun used for washing cars. There were $3-\mathrm{cm}$ lacerations in both the upper and lower eyelids of his left eye. The patient had left eye pain, visual disturbance, and left facial pain. Hypertension was the only item in his medical history, and his initial vital signs were stable. Tenderness and crepitus with palpation was observed on the left side of his face, neck, and shoulder. A facial/chest computed tomography (CT) scan revealed orbital emphysema, pneumomediastinum, and pneumocephalus, but no fractures were found (Figs. 1, 2). In

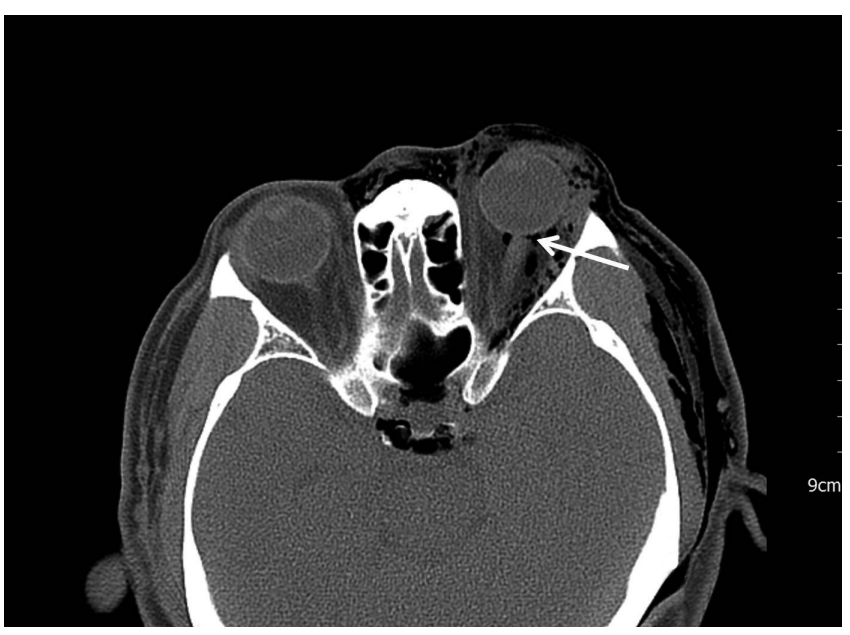

Fig. 1. Patient's facial computed tomography scan showing a nearly $70 \%$ transection of the optic nerve at the site of insertion to the eyeball (arrow). A fracture was not observed, and orbital emphysema extended to the parasellar area through the optic canal. addition, a partial optic nerve tear was observed near the insertion site of the left eyeball (Fig. 1). An eyesight test revealed deterioration of the vision in the left eye (finger count, $30 \mathrm{~cm}$; best-corrected visual acuity, 0.03) compared to the normal vision in the right eye (right eye visual acuity, 1.0). Intraocular pressure was 17 $\mathrm{mmHg}$ in the right eye and $22 \mathrm{mmHg}$ in the left eye. B-scan ultrasonography of both eyeballs showed unremarkable results.

Upon admission, primary closure was performed on the eyelid lacerations, and a lower canalicular repair was performed with a mini-Monoka stent insertion. Prophylactic antibiotics were prescribed. On the 4th day after admission, the patient was discharged. When the patient visited the ophthalmology department for a follow-up examination after 2 weeks, the finger count of his left eye was decreased to $20 \mathrm{~cm}$. In addition, the amplitude of the left P100 of the visual evoked potentials was significantly reduced, which indicated optic nerve injury (Fig. 3).

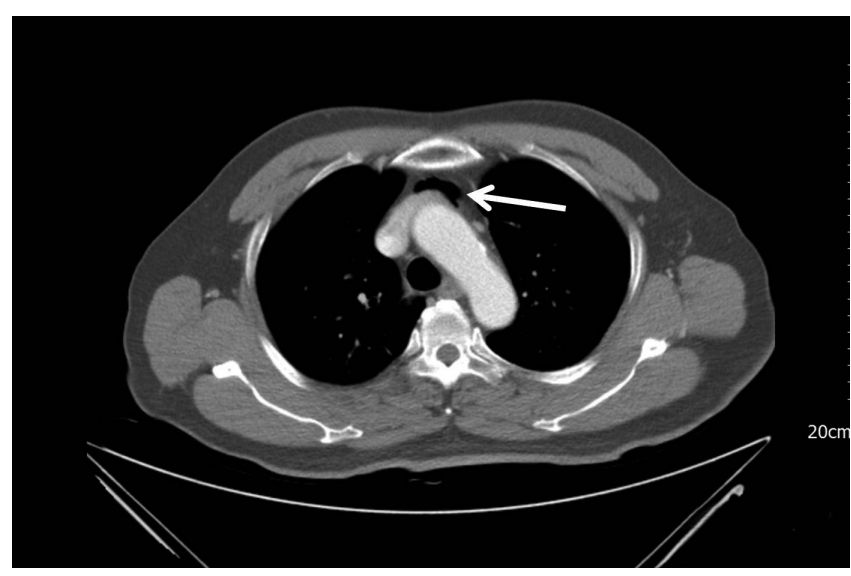

Fig. 2. Computed tomography of the patient's chest. A pneumomediastinum (arrow) is observed in the para-aortic arch.

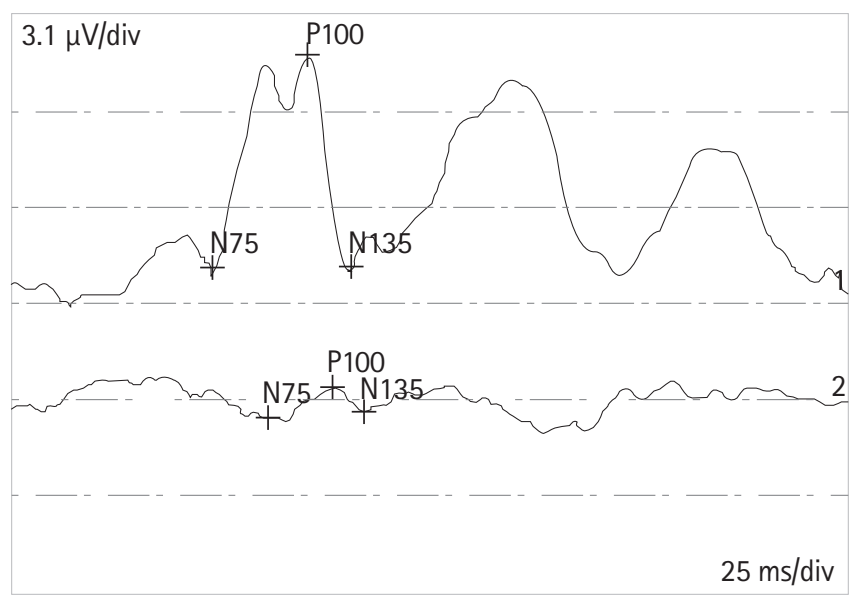

Fig. 3. Visual evoked potentials demonstrating a significant reduction in the P100 amplitude of the left eye. Line 1, visual evoked potentials of the right eye; line 2, visual evoked potentials of the left eye. 


\section{DISCUSSION}

High-pressure air is used in a variety of fields. However, when it is used inappropriately, it can cause orbital injury. The air pressure that causes the most orbital injuries is between 50 and 175 psi. $^{6}$ Green et al. ${ }^{9}$ reported that air pressure of at least $2.08 \mathrm{~J}$ caused an orbital fracture in a monkey.

Orbital emphysema occurs mostly with blowout fractures/paranasal sinus fractures. ${ }^{3,10}$ Orbital emphysema is rare in the absence of fractures, as was shown in this case. ${ }^{2,3}$ In the present case, a traumatic eyelid laceration that was followed by contact with high-pressure air resulted in subcutaneous emphysema in the face, neck, and shoulder with pneumomediastinum. The orbital emphysema and pneumocephalus were formed through conjunctival tissue, which is more loose than subcutaneous tissue. ${ }^{6}$ For the formation of a pneumocephalus, air passage within the cranium is thought to begin at the optic canal and travel to the cavernous sinus and then to the intracranial portion (Fig. 1). If there is subsequent exposure to high-pressure air, subcutaneous emphysema and pneumomediastinum can result, even when the external wound seems mild.

Optic nerve injuries can be divided into direct injuries and indirect injuries. ${ }^{11,12} A$ direct injury occurs when sharp objects, such as knives, penetrate the orbit and cause direct damage to the optic nerve, while an indirect injury results from nonpenetrating effects of trauma, such as hemorrhage, edema, and concussion. ${ }^{11,12}$ Because force that is applied to the superior orbital rim is passed on and concentrated to the orbital roof and canal, the intracanalicular optic nerve is the most common site of injury in traumatic optic neuropathy. ${ }^{13}$

In the current case, it was assumed that the most susceptible site, the insertion site of the intraorbital optic nerve, was disrupted due to the outward force of the eyeball from the increased intraorbital pressure that was initially induced by the high-pressure air. A nearly complete optic nerve separation at the insertion site to the eyeball was observed in the current patient's facial CT (Fig. 1). In most cases, optic nerve transection is a result of orbit disruption, which occurs typically as a result of fracture. ${ }^{11}$ However, pure soft tissue injuries can also cause optic nerve injuries. Thus, a meticulous review of a facial CT for evidence of optic nerve injury is important.

Because high-pressure air is used in various aspects of everyday life, related injuries are seen often in the emergency department. In general, orbital emphysema and pneumocephalus that are caused by high-pressure air show a relatively benign clinical course. However, when there is a temporary increase in intraor- bital pressure, as in cases that involve simple blunt trauma, highvelocity trauma, blast injury, and so forth, optic nerve injury cannot be ruled out, and, consequently, the possibility of permanent visual deterioration remains. Thus, emergency physicians should look for apparent or subtle optic nerve injury when treating such patients, as this can have a detrimental impact on the patients' prognoses.

\section{CONFLICT OF INTEREST}

No potential conflict of interest relevant to this article was reported.

\section{REFERENCES}

1. Hwang $\mathrm{K}$, Kim DH, Lee HS. Orbital fracture due to high-pressure air injection. J Craniofac Surg 2011;22:1506-7.

2. Yuksel M, Yuksel KZ, Ozdemir G, Ugur T. Bilateral orbital emphysema and pneumocephalus as a result of accidental compressed air exposure. Emerg Radiol 2007;13:195-8.

3. Caesar R, Gajus M, Davies R. Compressed air injury of the orbit in the absence of external trauma. Eye (Lond) 2003;17:661-2.

4. Stroh EM, Finger PT. Traumatic transconjunctival orbital emphysema. Br J Ophthalmol 1990;74:380-1.

5. Williams TR, Frankel N. Intracerebral air caused by conjunctival laceration with air hose. Arch Ophthalmol 1999;117:1090-1.

6. Hiraoka T, Ogami T, Okamoto F, Oshika T. Compressed air blast injury with palpebral, orbital, facial, cervical, and mediastinal emphysema through an eyelid laceration: a case report and review of literature. BMC Ophthalmol 2013;13:68.

7. Mathew S, Vasu U, Francis F, Nazareth C. Transconjunctival orbital emphysema caused by compressed air injury: a case report. Indian J Ophthalmol 2008;56:247-9.

8. Gross JG, Doxanas MT. Traumatic optic atrophy caused by compressed air. Ann Ophthalmol 1987;19:69-70,74.

9. Green RP Jr, Peters DR, Shore JW, Fanton JW, Davis H. Force necessary to fracture the orbital floor. Ophthal Plast Reconstr Surg 1990;6:211-7.

10. Carter KD, Nerad JA. Fluctuating visual loss secondary to orbital emphysema. Am J Ophthalmol 1987;104:664-5.

11. Sarkies N. Traumatic optic neuropathy. Eye (Lond) 2004;18: 1122-5.

12. Steinsapir KD, Goldberg RA. Traumatic optic neuropathy: an evolving understanding. Am J Ophthalmol 2011;151:928-33.e2.

13. Kumaran AM, Sundar G, Chye LT. Traumatic optic neuropathy: a review. Craniomaxillofac Trauma Reconstr 2015;8:31-41. 